\title{
Menger Curvature Based 3D Printing Watermarking Algorithm
}

\author{
Giao N. Pham ${ }^{1}$, Suk-Hwan Lee ${ }^{2}$, Eung-Joo Lee ${ }^{3}$ and Ki-Ryong Kwon ${ }^{1^{*}}$ \\ ${ }^{1}$ Dept. of IT Convergence \& Application Engineering, Pukyong National \\ University, Pusan, South Korea \\ ${ }^{2}$ Dept. of Information Security, ${ }^{3}$ Dept. of Information Communication \\ Engineering, Tongmyong University, Pusan, South Korea \\ ngocgiaofet@gmail.com,skylee@tu.ac.kr,ejlee@tu.ac.kr,krkwon@pknu.ac.kr
}

\begin{abstract}
Nowadays, 3D printing technology can change the world based on the fact that it enhances the creation of complex structures and shapes that were not feasible in the past. So $3 D$ printing data is often illegally used without permission from the original providers. This paper presents a novel watermarking algorithm for the copyright protection of $3 D$ printing data based on the Menger curvature. Facets of $3 D$ printing data are classified into groups based on the Menger curvature and the mean Menger curvature of each group will be then computed in order to embed watermark data. The watermark data is embedded to the groups of facets by changing the mean Menger curvature of each group based on the reference of special Menger curvature. In each group, we select a facet which has the nearest Menger curvature with the changed mean Menger curvature and change the vertices of the selected facet according to the changed Menger curvature for the watermarked $3 D$ printing data generation. In experiments, the distance error between the original $3 D$ printing file and the watermarked $3 D$ printing file is approximate zero, and the Bit Error Rate is also very low. From experimental results, we verify that the proposed method is invisible and robustness with geometric attacks as rotation, scaling and translation.
\end{abstract}

Keywords: $3 D$ printing copyright, 3D model watermarking, 3D slicing, Additive Layer Manufacturing

\section{Introduction}

$3 \mathrm{D}$ printing is a process of creating objects directly by adding material layer by layer in a variety of ways $[1,2]$. Thus, 3D printing technology allows users to turn any digital file into a three dimensional physical product. 3D printing can be essential in improving the quality of life of individuals whose organs have failed. In addition, the applications of 3D printing in the context of health has the potential of increasing the life expectancy of humans in that it saves countless lives that are on the verge of death. Summary, it turns users from being passive consumers to active creators.

Due to the benefits of $3 \mathrm{D}$ printing is enormous in all domains and the price of a $3 \mathrm{D}$ printer is not expensive, individual users can buy a 3D printer and download 3D models on Internet to print real objects out. This makes a large effect on manufacturers, and they need a copyright protection solution for 3D printing. Moreover, the original providers also desire to identify their products for commercial transactions. So, a watermarking solution for 3D printing is necessary for the ownership and copyright protection [3].

Previously, there are many watermarking methods for 3D contents, 3D mesh and 3D animals. But these methods are only useful for copyright protection related to $3 \mathrm{D}$

Received (April 6, 2017), Review Result (November 6, 2017), Accepted (November 8, 2017)

* Corresponding Author 
contents. They could not be applied to 3D printing. Because the output of 3D printing is a real object. The purpose of $3 \mathrm{D}$ printing watermarking is how to extract the embedded watermark data from the scanned model of 3D printed object. For responding to the issues related to the copyright protection of $3 \mathrm{D}$ printing, we proposed a novel watermarking algorithm for $3 \mathrm{D}$ printing in this paper. The main points of the proposed algorithm is to classify facets into groups based on the Menger curvature of facet before embedding watermark data to the groups of facets. The number of groups is dynamic and defined by user. The watermark bit is embedded by changing the mean Menger curvature of group on the reference of special value. The watermark data is extracted from the scanned model of $3 \mathrm{D}$ printed object.

To clarify the proposed algorithm, we organized our paper as follow: in Section 2, we look into previous watermarking techniques for 3D model, and explain the relation of Menger curvature to the proposed algorithm. In Section 3, we show the proposed algorithm in detail. Experimental results and the evaluation of the proposed algorithm will be shown in Section 4. Section 5 shows the conclusion.

\section{Related Work}

\subsection{D Model Watermarking}

3D model watermarking has been extensively researched since the early 2000s. 3D watermarking schemes are generally focused on geospatial domain and frequency domain [4-17]. The main concept of watermarking methods in the geospatial domain is to embed the watermark by modifying the value of vertices or geometric features as length, area or topology features while the main concept of watermarking schemes in the frequency domain is to embed the watermark in the spectrum coefficients of DFT, DWT, and DCT of a sequence of vertices or topologies. Thus, these methods only generated the watermarked 3D models, and they are then used again for watermark extraction. It is not the end purpose of 3D printing watermarking. The end purpose of $3 \mathrm{D}$ printing watermarking is to extract the embedded watermark data from the 3D printed object of the watermarked 3D models. So the proposed schemes above cannot apply to the purpose of 3D printing watermarking.

S. Yamazaki et al. [18] proposed a method of extracting watermark from 3D prints created from 3D mesh data. The watermark is embedded to the 3D mesh based on the spread spectrum technique, and then extracted from $3 \mathrm{D}$ prints by reconstructing the $3 \mathrm{D}$ mesh homologous to the original. The accuracy of this method is not high. Because, the scanned 3D model has many noises in the scanning process and the spread spectrum was changed a lot after transformation.

\subsection{Menger Curvature Based 3D Printing Watermarking}

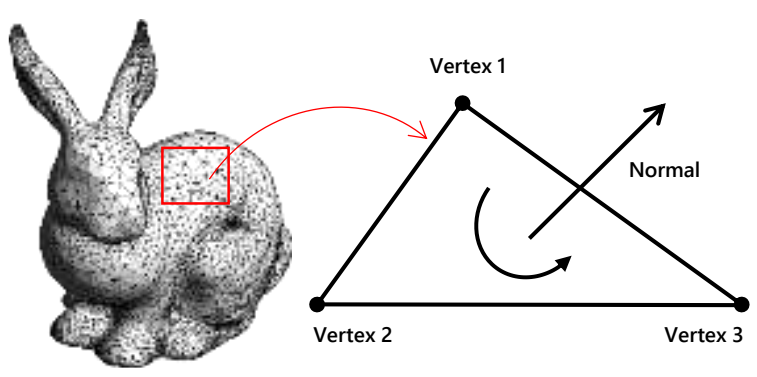

(a)

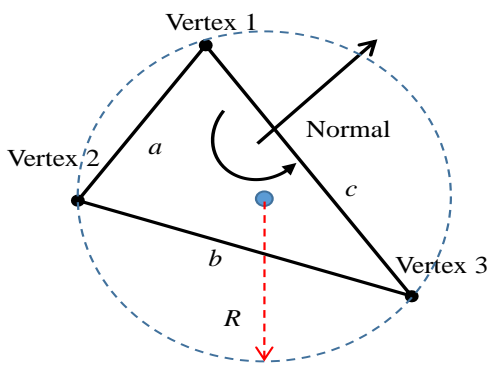

(b)

Figure 1. (a) Structure of Facet and (b) Circumscribed Circle of Facet 
The input of 3D printing is a 3D triangle mesh [19], which is designed by a CAD software. A 3D triangle mesh contains a set of facets. Each facet includes three vertices and a normal vector. Each vertex is presented by three coordinates $\mathrm{x}, \mathrm{y}$ and z. Figure 1 shows the structure of a facet. The Menger curvature is the curvature of a triple of points in n-dimensional Euclidean space [20,21]. Due to the fact that a 3D triangle mesh includes a set of facets. Each facet is a triple of points. So, the Menger curvature of a facet is computed by Eq. (1) below:

$K_{M}=\frac{1}{R}=\frac{4 \cdot A}{a \cdot b \cdot c}$

With $K_{M}$ is the Menger curvature of facet, $A$ is the area of facet, $R$ is the circumscribed circle radius of facet, $a, b$ and $c$ are the edge of facet respectively.

From Eq. (1), we can see that the Menger curvature of a facet is dependent on the circumscribed circle radius of that facet or the length of edges of that facet. After the scanning of $3 \mathrm{D}$ printed object and reconstruction, we will get the scanned 3D triangle mesh. Due to the effect of noise in the scanning process, the coordinates of vertices of each triangle in the scanned 3D triangle mesh is not the same with original 3D triangle mesh but the overall shape of facets is not change. Due to the shape of facet is not change, the Menger curvature of facet is also not change. So, we could use the Menger curvature of facet for watermarking.

\section{The Proposed Algorithm}

\subsection{Overview}

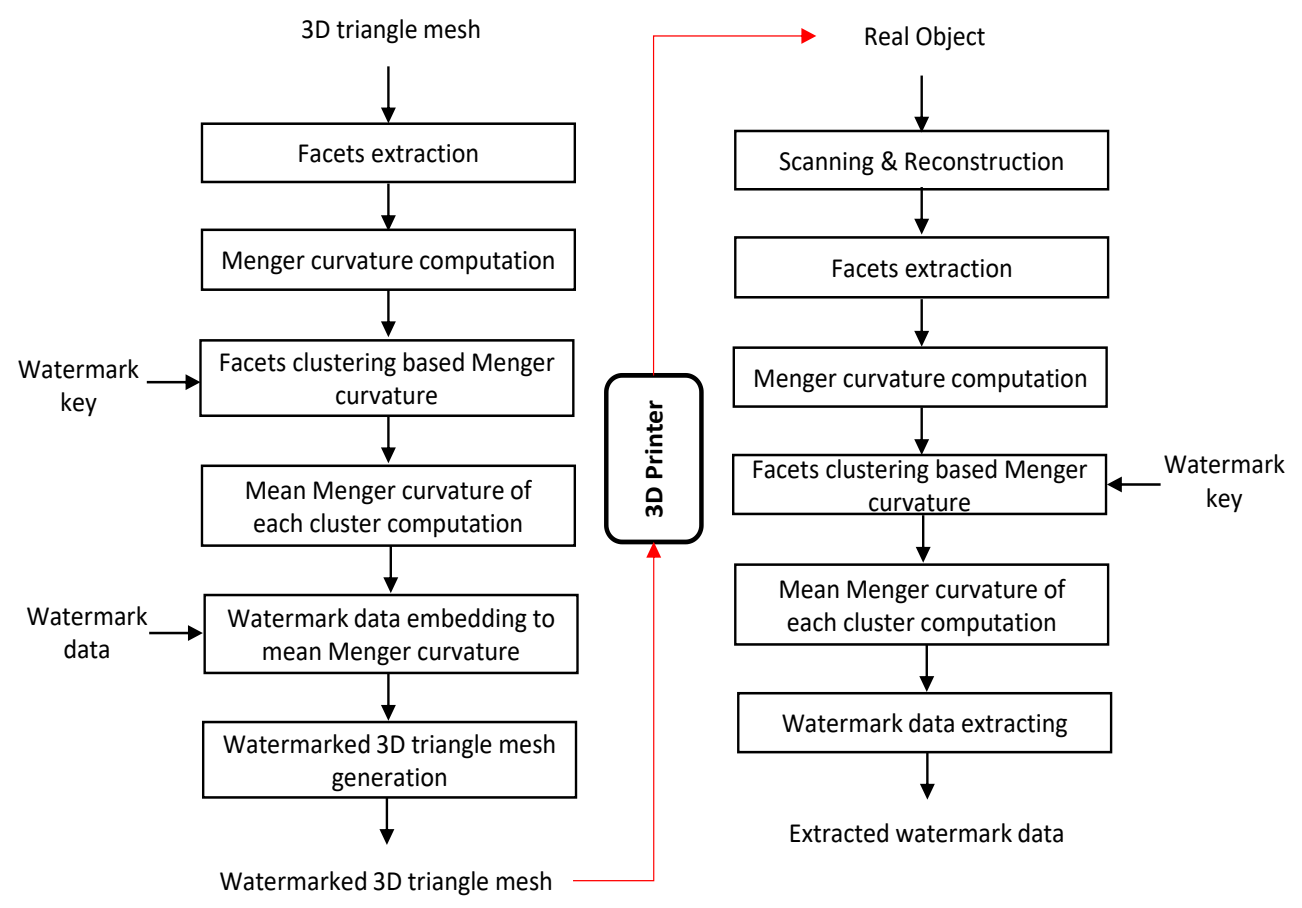

Figure 2. The Proposed Algorithm

The proposed algorithm is described in Figure 2. Facets are extracted from 3D printing file (3D triangle mesh) to compute their Menger curvature. After that, facets are classified into groups by the clustering algorithm based on their Menger curvature. Watermark key is the number of groups that we want to classify facets. 
With each group of facets, we compute the mean Menger curvature of it, and embed a watermark bit to that mean Menger curvature. The watermarked 3D printing file is generated according to mean Menger curvatures which have been embedded watermark data.

After printing, the real object will be used for scanning and reconstruction to generate the scanned 3D printing file (the scanned 3D triangle mesh). Then, facets are extracted from the scanned 3D printing file for computing Menger curvature and clustering facets into groups. Watermarking data is extracted from the mean Menger curvature of each group. We will describe the detailed watermark embedding and extracting processes in Section 3.2 and Section 3.3.

\subsection{Watermark Embedding}

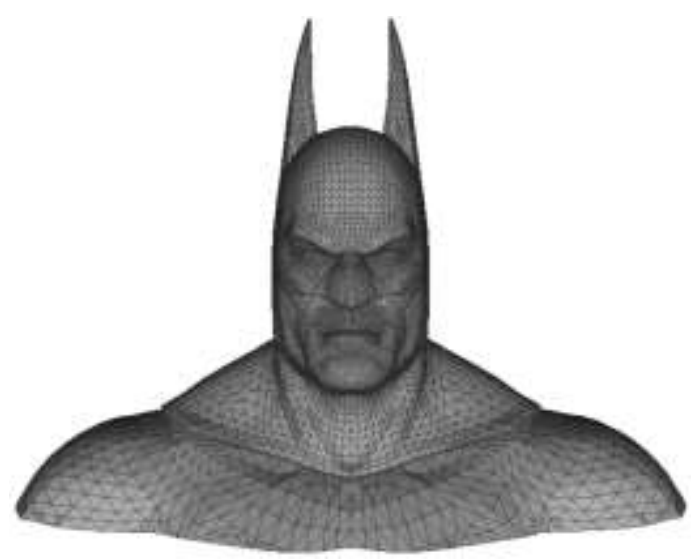

(a)

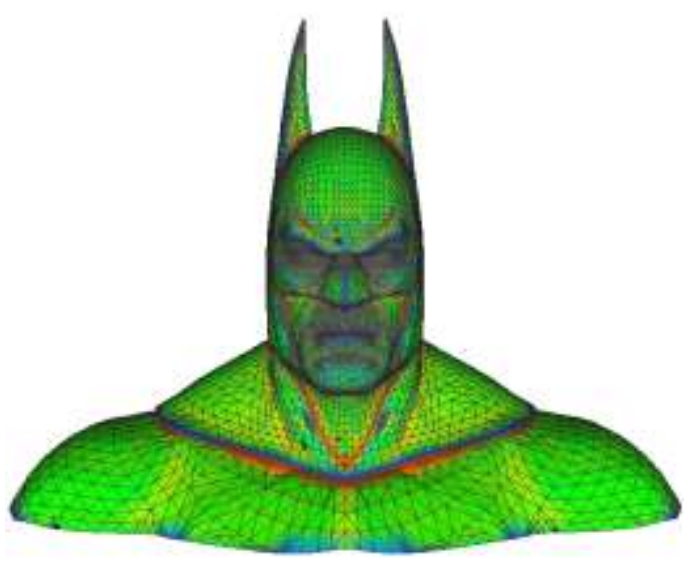

(b)

Figure 3. (a) Original Batman Mesh, and (b) Facets Clustering based on Menger Curvature

A 3D printing file contains a number of facets, $\mathbf{F}=\left\{f_{i} \mid i \in[1, N]\right\}$. Each facet contains three vertices (three points), $f_{i}=\left\{v_{i j} \mid j \in[1,3]\right\}$. The Menger curvature of each facet $f_{i}$ is computed by its vertices and corresponding area:

$$
K_{i}=\frac{4 A}{\left|v_{i 1}-v_{i 2}\right| \cdot\left|v_{i 2}-v_{i 3}\right| \cdot\left|v_{i 3}-v_{i 1}\right|}
$$

Therein $A$ is the area of facet. $N$ facets are divided into $M$ groups by the clustering algorithm based on the value of Menger curvature, $M=\left\{m_{g} \mid g \in[1, M]\right\}$. Figure 3 shows the result of facets clustering of Batman mesh based on the Menger curvature.

After classifying facets into $M$ groups, we find the maximum Menger curvature, minimum Menger curvature of each group, and calculate the mean Menger curvature of each group. Assume that $K_{\text {max }}^{m_{g}}, K_{\text {min }}^{m_{g}}$ and $K_{\text {mean }}^{m_{g}}$ are the maximum Menger curvature, minimum Menger curvature and mean Menger curvature of group $m_{g}$ respectively. The mean Menger curvature $K_{\text {mean }}^{m_{g}}$ of group $m_{g}$ is the average value of all Menger curvatures in group, with $\left|m_{g}\right|$ is the number of facets in group $m_{g}$ :

$K_{\text {mean }}^{m_{g}}=\frac{\sum K_{i} \in m_{g}}{\left|m_{g}\right|}$

Next, we define $\Delta_{m g}$ is the average value of $K_{\max }^{m_{g}}$ and $K_{\min }^{m_{g}}$ : 
$\Delta_{m g}=\frac{K_{\min }^{m_{g}}+K_{\max }^{m_{g}}}{2}$

And each of watermark bits $\omega_{g} \in\{0,1\}(g \in[1, M])$ is embedded by changing the mean Menger curvature of group $m_{g}$ on the reference of the average value $\Delta_{m g}$. If $\omega_{g}=$ 0 , then $K_{\text {mean }}^{m_{g}}$ will be move to be less than $\Delta_{m g}$. If $\omega_{g}=1$, then $K_{\text {mean }}^{m_{g}}$ will be move to be greater than $\Delta_{m g}$ :

$$
K_{\text {mean }}^{m_{g^{*}}}=\left\{\begin{array}{l}
K_{\text {mean }}^{m_{g^{*}}}>\Delta_{m g} \text { if } \omega_{g}=1 \\
K_{\text {mean }}^{m_{g^{*}}}<\Delta_{m g} \text { if } \omega_{g}=0
\end{array}\right.
$$

For satisfying the above embedding condition, the watermarked mean distance $K_{\text {mean }}^{m_{g^{*}}}$ will be changed as follows:

$$
\begin{aligned}
& \text { If } \omega_{g}=1, K_{\text {mean }}^{m_{g^{*}}}=\left\{\begin{array}{c}
\Delta_{m g}+\frac{\Delta_{m g}-K_{\text {mean }}^{m_{g}}}{2} \text { if } K_{\text {mean }}^{m_{g}}<\Delta_{m g} \\
\text { No change if } K_{\text {mean }}^{m_{g}} \geq \Delta_{m g}
\end{array}\right. \\
& \text { If } \omega_{g}=0, K_{\text {mean }}^{m_{g^{*}}}=\left\{\begin{array}{c}
\Delta_{m g}-\frac{K_{\text {max }}^{m_{g}}-K_{\text {mean }}^{m_{g}}}{4} \text { if } K_{\text {mean }}^{m_{g}} \geq \Delta_{m g} \\
\text { No change if } K_{\text {mean }}^{m_{g}}<\Delta_{m g}
\end{array}\right.
\end{aligned}
$$

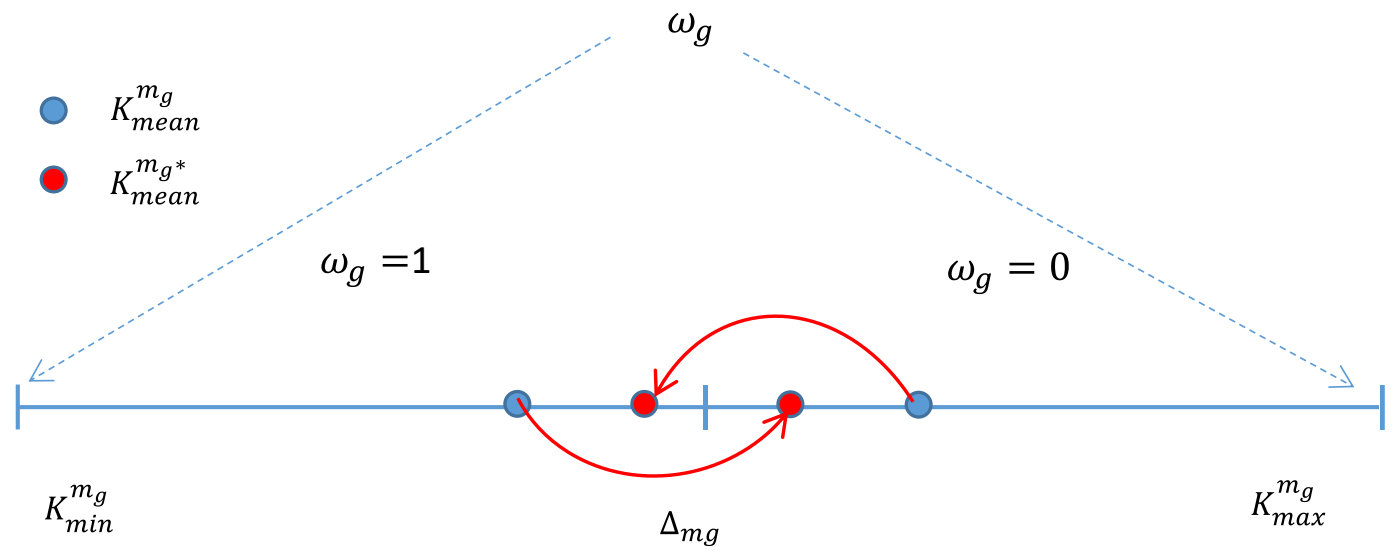

\section{Figure 4. Watermark Bit Embedding by Changing the Mean Menger Curvature}

Figure 4 shows the change of the mean Menger curvature $K_{\text {mean }}^{m_{g}}$ of group $m_{g}$ according to the watermark bit $\omega_{g}$. The mean Menger curvature $K_{\text {mean }}^{m_{g}}$ is represented by blue point. The watermarked mean Menger curvature $K_{\text {mean }}^{m_{g^{*}}}$ is represented by red point. When $\omega_{g}=0, K_{\text {mean }}^{m_{g}}$ will be move to be less than $\Delta_{m g}$ if it is equal or greater than $\Delta_{m g}$. When $\omega_{g}=1, K_{\text {mean }}^{m_{g}}$ will be move to be greater than $\Delta_{m g}$ if it is less than $\Delta_{m g}$.

After embedding watermark bit $\omega_{g}$ to the mean Menger curvature of group $m_{g}$, the change rate $\alpha_{g}$ is calculated as depicted in Eq. (8): 


$$
\alpha_{g}=\frac{K_{m e a n}^{m_{g^{*}}}}{\Delta_{m g}}
$$

The change rate $\alpha_{g}$ is used to change the vertices of a facet in group $m_{g}$. The Menger curvature of this facet must be the nearest value with the watermarked mean Menger curvature $K_{\text {mean }}^{m_{g^{*}}}$. Assume that, if $f_{i}$ is a facet in group $m_{g}$ and its Menger curvature is the nearest value with the watermarked mean Menger curvature $K_{\text {mean }}^{m_{g^{*}}}$, then the vertices of $f_{i}$ is changed by the change rate $\alpha_{g}$ as follow:

$$
v_{i j}^{\prime}=\alpha_{g} \cdot v_{i j}+\left(\alpha_{g}-1\right) \cdot v_{i j} \forall j \in[1,3]
$$

Where $v_{i j}^{\prime} \mid j \in[1,3]$ are the new vertices of facet $f_{i}$ after changing by Eq. (9).

\subsection{Watermark Extracting}

The watermark extraction process is similar the embedding process. Firstly, we also extract facets from the scanned 3D printing file to compute the Menger curvature of facets. After that, we classify them into groups by the clustering algorithm based on the Menger curvature. The watermark key is used for the clustering process. For each group, we find the maximum Menger curvature $K_{\max }^{m_{g^{\prime}}}$, the minimum Menger curvature $K_{\text {min }}^{m_{g^{\prime}}}$, and calculate the mean Menger curvature $K_{\text {mean }}^{m_{g^{\prime}}}$ similar Eq. (3). And $\Delta_{m g}^{\prime}=\left(K_{m i n}^{m_{g^{\prime}}}+\right.$ $\left.K_{\max }^{m_{g^{\prime}}}\right) / 2$ is the average value of $K_{\min }^{m_{g^{\prime}}}, K_{\max }^{m_{g^{\prime}}}$. Finally, the watermark bit $\omega_{g}$ can be extracted by comparing the mean Menger curvature $K_{\text {mean }}^{m_{g^{\prime}}}$ and the average value $\Delta^{\prime}{ }_{m g}$ :

$\omega_{g}=\left\{\begin{array}{l}1 \text { if } K_{\text {mean }}^{m_{g^{\prime}}} \geq \Delta^{\prime}{ }_{m g} \\ 0 \text { if } K_{\text {mean }}^{m_{g^{\prime}}}<\Delta^{\prime}{ }_{m g}\end{array}\right.$

\section{Experimental Results and Analysis}

Table 1. Experimental Results

\begin{tabular}{|l|l|l|l|l|}
\hline Name & $\begin{array}{l}\text { \# of } \\
\text { facets }\end{array}$ & \# of groups & $\begin{array}{l}\text { Mean distance } \\
\text { error }\end{array}$ & BER (\%) \\
\hline Sword & 2146 & 67 & $9.965999 \mathrm{E}-06$ & 0.0000 \\
\hline Tower & 3202 & 100 & $9.98818 \mathrm{E}-06$ & 0.0100 \\
\hline Airplane & 9052 & 282 & $9.994756 \mathrm{E}-06$ & 0.0106 \\
\hline Batman & 13566 & 339 & $9.98718 \mathrm{E}-06$ & 0.0353 \\
\hline Batman Armor & 29496 & 737 & $9.989746 \mathrm{E}-06$ & 0.0081 \\
\hline Gun & 33072 & 826 & $9.993756 \mathrm{E}-06$ & 0.0147 \\
\hline Rocket & 63497 & 1587 & $9.999013 \mathrm{E}-06$ & 0.0242 \\
\hline
\end{tabular}

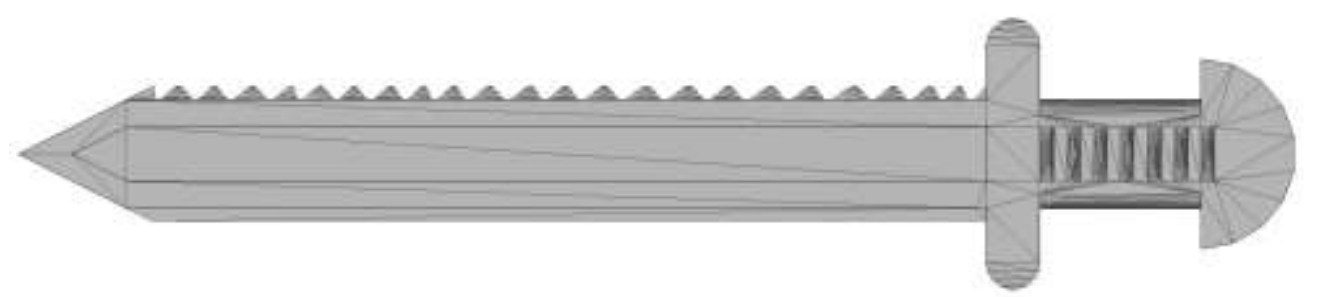

Sword 

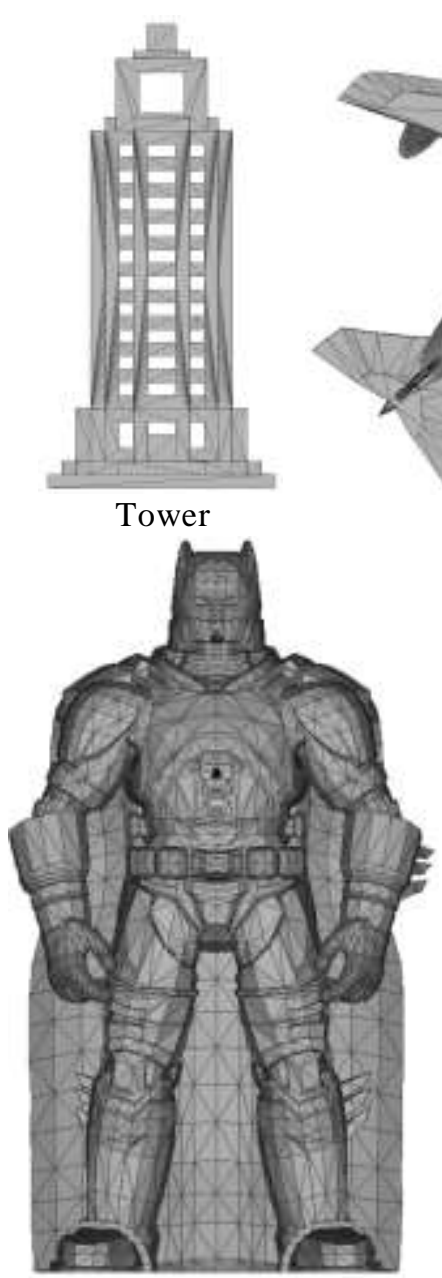

Batman Amor

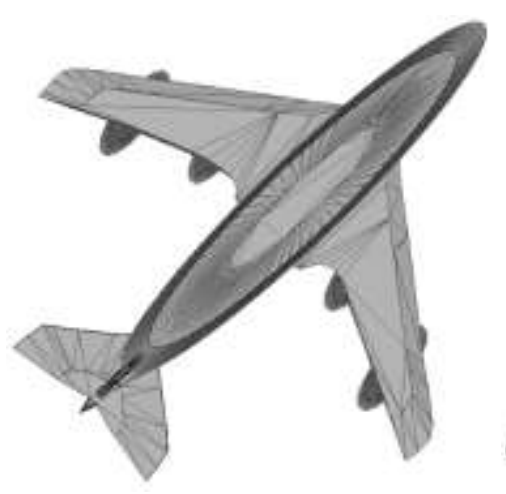

Airplane

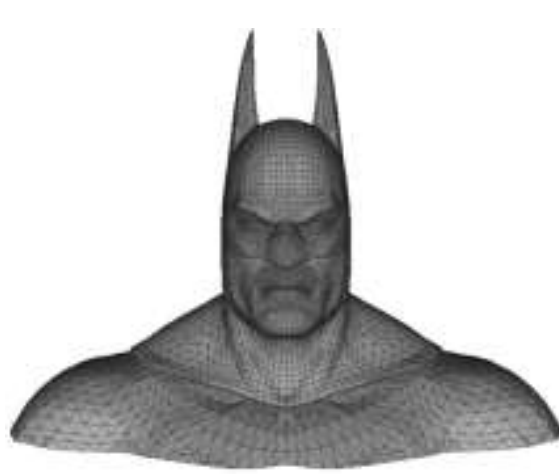

Batman

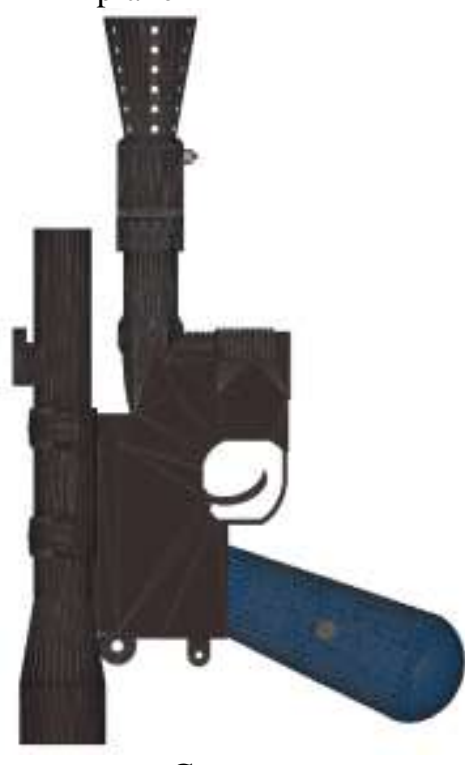

Gun

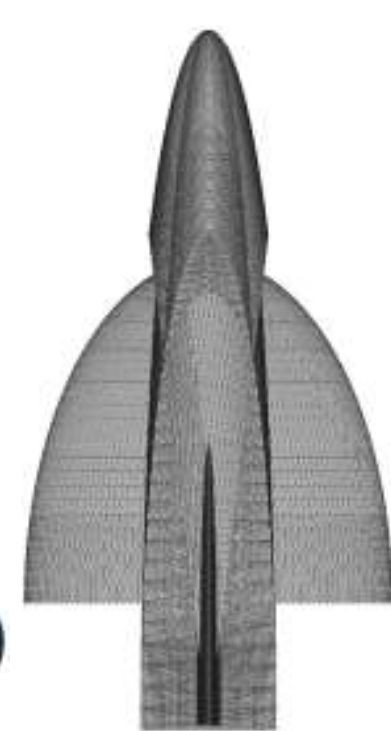

Rocket

Figure 5. Test Models

We experimented the proposed algorithm with test models in Figure 5. The format of models is STL file. The detailed information of models is shown in Table1. Test models are embedded watermark data according to the watermark key. The watermark key is the number of groups which is defined by user, and used for clustering facets. We used K-mean algorithm for clustering facets [23]. The number of groups is always less than a haft of facets. To safety above condition, we defined the number of groups $M$ according to the number of facets $N$ as Eq. (11).

$M=$ Integer $\operatorname{part}\left(\frac{N}{2^{3} \times \operatorname{Sum}}\right)$

With Sum is the number of digits of $N$. For example, if $N=2146$ then Sum $=4$. The length of watermark bit is equal the number of groups. In order to evaluate the performance of the proposed algorithm, we compute the data accuracy and evaluate the robustness of the proposed algorithm.

\subsection{Data Accuracy}

We compute data accuracy to evaluate the invisibility of the proposed algorithm. We calculated the mean Euclidean distance error $d^{m}\left(v, v^{\prime}\right)$ between the vertices $v^{\prime}$ of the watermarked 3D printing file and the vertices $v$ of the original 3D printing file to analyze 
the vertices data accuracy and invisibility. The mean Euclidean distance error $d^{m}\left(v, v^{\prime}\right)$ is the mean distance error of all distances and calculate by Eq. (12).

$d^{m}\left(v, v^{\prime}\right)=\frac{1}{3 \times \mathrm{N}} \sum_{i=1}^{\mathrm{N}} \sum_{j=1}^{3}\left\|v_{i j}-v^{\prime}{ }_{i j}\right\|$

Table 1 shows the experimental results with respect to data accuracy. We experimented test models with differential watermarks. The mean distance error between the original $3 \mathrm{D}$ printing file and the watermarked $3 \mathrm{D}$ printing file is very small. The mean distance error is dependent on the number of the watermarked vertices. The number of the watermarked vertices is dependent on the number of groups, the mean Menger curvature of each group and the clustering algorithm. From Table 1, the mean distance error seems increase according to the number of groups. The distance error is varied from $9.965 \mathrm{E}-6$ to 9.999E-6. It means the change rate of the watermarked 3D printing file is very small. So, the invisibility of the proposed algorithm is very high. Figure 6 shows the mean distance error of test models according to the number of groups.

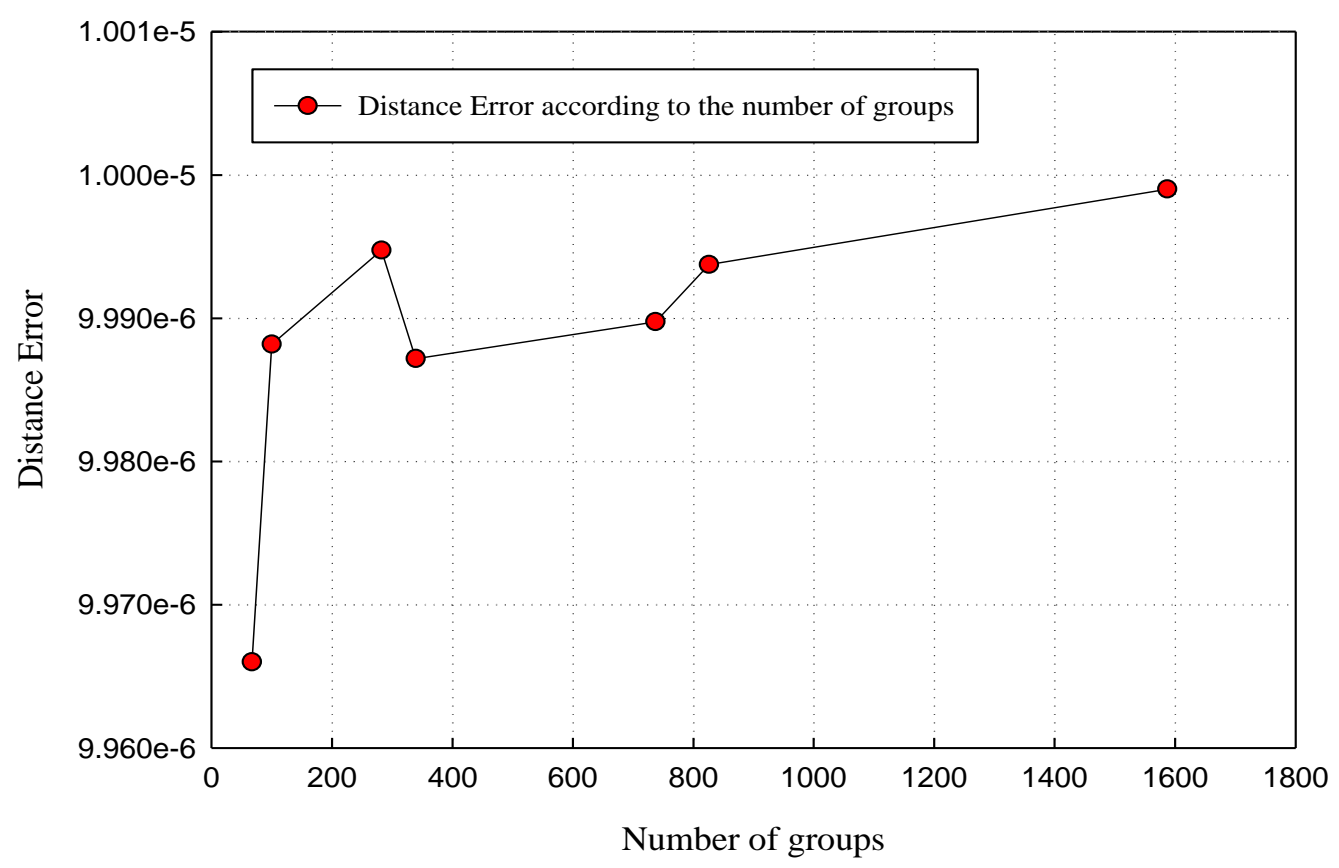

Figure 6. Distance Error According To the Number of Groups

\subsection{Robustness Evaluation}

Due to the scanned 3D printing file is affected by noise in the scanning process. Thus, it is not perfectly the same with the original 3D printing file. Due to translation and rotation only change the spatial location of 3D printing file. Thus, we only re-scale the scanned 3D printing file before extracting facets for watermark extraction. In order to evaluate the robustness of the proposed algorithm, we calculate the bit error rate (BER). We calculate BER by comparing the extracted watermark data with the original watermark data as shown in Eq. 13.

$B E R=\frac{\text { Extracted } \text { watermark }}{\text { Original } \text { watermark }} \times 100 \%$

Experiments showed that the BER is very low. It means the proposed algorithm is robustness with geometric attacks. The BER is dependent on the number of the 
watermarked facets. The number of the watermarked facets is dependent on the number of groups, the number of facets in each groups and the clustering algorithm. The BER is varied from $0.000 \%$ to $0.0353 \%$. Figure 7 shows the BER of the proposed algorithm according to the number of groups. The BER seems increase according to the number of groups.

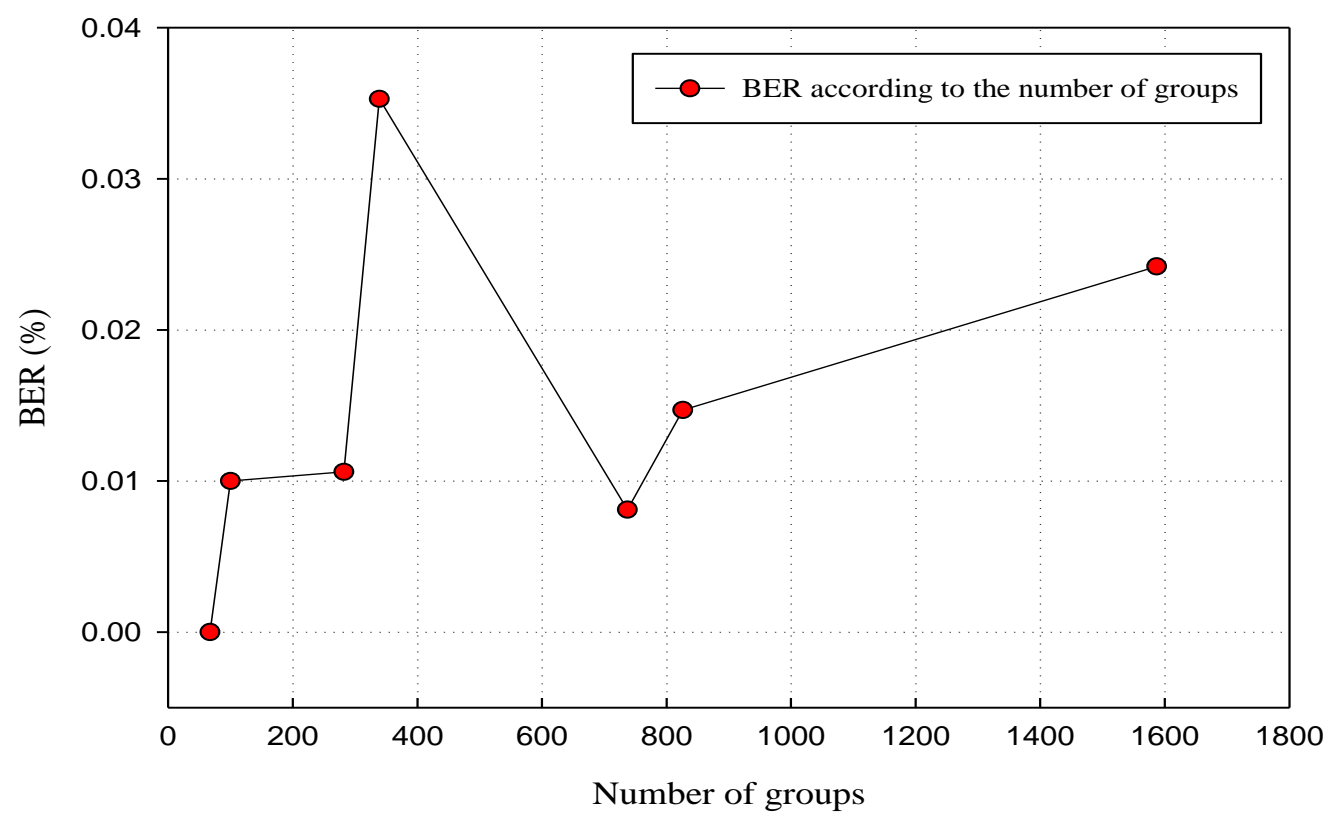

Figure 7. The BER According to the Number of Groups

\section{Conclusion}

In this paper, we proposed a novel watermarking algorithm for 3D printing. It is based on the Menger curvature of facets and the clustering algorithm. We experimented the proposed algorithm with many 3D printing files. Experimental results showed that the mean distance error between the watermarked 3D printing file and the original 3D printing files is very small. Experimental results verified that the proposed method has low BER. This mean the proposed algorithm is invisibility and robustness with geometric attacks as rotation, translation and scaling. Next time, we will improve the proposed algorithm and apply to the copyright protection system.

\section{Acknowledgments}

This work is supported by the by Basic Science Research Program through the National Research Foundation of Korea(NRF) funded by the Ministry of Science and ICT (No. 2016R1D1A3B03931003, No. 2017R1A2B2012456), Institute for Information \& communications Technology Promotion(IITP) grant funded by the Korea government (MSIT)(No.2015-0-00225), and the MSIP(Ministry of Science and ICT), Korea, under the Grand Information Technology Research Center support program (IITP-2017-2016-000318) supervised by the IITP(Institute for Information \& communications Technology Promotion)".

\section{References}

[1] United States Government Accountability Office, "3D Printing Opportunities, Challenges, and Policy Implications of Additive Manufacturing”, USA, (2015).

[2] 3D Systems Circle Rock Hill, "White paper: How 3D Printing works, The Vision, Innovation and Technologies behind Inkjet 3D Printing", (2012). 
[3] S. Ira and S. Parker, "Copyright Issues in 3D Printing", Proceeding of the International Technology Law Conference, Paris, France, (2014).

[4] Q. Ai, Q. Liu, D. Zhou, L. Yang and Q. Xi, "A new digital watermarking scheme for 3D triangular mesh models", Journal of Signal Processing, vol. 89, (2009), pp. 2159-2170.

[5] E. Zagrouba and B. Jabra, "A New Approach of Mesh Watermarking Based on Maximally Stable Meshes Detection", Proceedings of the 3rd international conference on New technologies, Mobility and Security, Cairo, Egypt, (2009).

[6] K. Wang, L. Ming, G. Adrian and D. Florence, "Blind and robust mesh watermarking using manifold harmonics", Proceeding of 16th IEEE International Conference on Image Processing, Cairo, Egypt, (2009).

[7] L. Yang, P. Balakrishnan and G.H Xiao, "Blind invisible watermarking for 3D meshes with textures", Proceeding of The 17th IEEE International Conference on Image Processing, Hongkong, (2010).

[8] M. Mona, A. Ella and O. Hoda, "Robust Watermarking Approach for 3D Triangular Mesh using Self Organization Map", Proceeding of 8th International Conference on Computer Engineering \& Systems, Cairo, Egypt, (2013).

[9] X-H Tan, "A 3D Model Asymmetric Watermarking Algorithm Based on Optimization Statistics", Journal of Theoretical and Applied Information Technology, vol. 51, no. 2, (2013), pp. 175-181.

[10] P. Ramya and Dr. Nalini, "A New Watermarking For 3D Models Based On Integral Invariants", International Journal of Innovative Research in Computer and Communication Engineering, vol. 1, no. 2, (2013), pp. 190-192.

[11] C. Tamane, and R. Ratnadeep, "Blind 3D Model Watermarking Based on Multi-Resolution Representation and Fuzzy Logic", International Journal of Computer Science \& Information Technology, vol. 4, no. 1, (2012), pp. 117-126.

[12] X. Feng, Y. Liu and L. Fang, "Digital Watermark of 3D CAD Product Model", International Journal of Security and Its Applications, vol. 9, no. 9, (2015), pp. 305-320.

[13] J. Wang, Y. Chang, Y. Chun and S. Yu, "Hamming Code Based Watermarking Scheme for 3D Model Verification", Proceeding of 2014 International Symposium on Computer, Consumer and Control, Taichung, Taiwan, (2014).

[14] J. Liu, Y. Wang, W. He and Y. Li, “A New Watermarking Method of 3D Mesh Model”, Indonesian Journal of Electrical Engineering, vol. 12, no. 2, (2014), pp. 1610-1617.

[15] X. Rolland, G. Do and A. Pierre, "Triangle Surface Mesh Watermarking based on a Constrained Optimization Framework", IEEE Transactions on Information Forensics and Security, vol. 9, no. 9, (2014), pp. 1491-1501.

[16] Q. Hu and Z. Lang, "The Study of 3D Digital Watermarking Algorithm Which is based on a Set of Complete System of Legendre Orthogonal Function", The Open Automation and Control Systems Journal, vol. 6, (2014), pp. 1710-1716.

[17] J.U Ho, D.G Kim, S.H Choi and H.K Lee, "3D Print-Scan Resilient Watermarking Using a HistogramBased Circular Shift Coding Structure", Proceedings of the 3rd ACM Workshop on Information Hiding and Multimedia Security, Portland, USA, (2015).

[18] S. Yamazaki, K. Satoshi and M. Masaaki, "Extracting Watermark from 3D Prints", The 22nd International Conference on Pattern Recognition, Stockholm, Sweden, (2015).

[19] STL format in 3D printing, https://all3dp.com/what-is-stl-file-format-extension-3d-printing/, Accessed, (2016).

[20] Lecture Notes in Mathematics, "Analytic Capacity, Rectifiability, Menger Curvature and the Cauchy Integral," Springer, vol. 1799, (2004).

[21] F. Leymarie, "Notes on Menger Curvature", retrieved November, (2007).

[22] M. Queen, "K-means clustering algorithm", Some Methods for Classification and Analysis of Multivariate Observations, University of California Press, Berkeley, Calif., vol. 1, (1967), pp. 281-297.

\section{Authors}

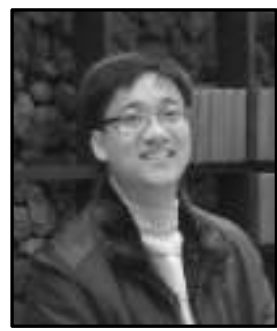

Giao N. Pham, he received the Degree of Engineering in School of Electronic \& Telecommunication in Hanoi University of Science \& Technology (HUST) in 2011, and Master degree from Pukyong National University (PKNU), Busan, South Korea in 2014. Currently, he is Ph.D candidate in PKNU. His research interests include digital image processing application, GIS visualization, multimedia data security, IoT and smart systems. 


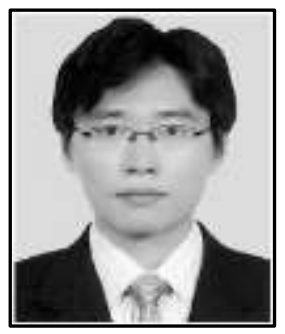

Suk-Hwan Lee, he received a B.S., a M.S., and a Ph.D. Degrees in Electrical Engineering from Kyungpook National University, Korea in 1999, 2001, and 2004 respectively. He is currently an associate professor in Department of Information Security at Tongmyong University. His research interests include multimedia security, digital image processing, and computer graphics.

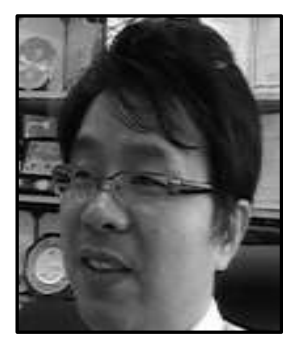

Eung-Joo Lee, he received his B. S. M. S. and Ph. D. in Electronic Engineering from Kyungpook National University, Korea, in 1990, 1992, and Aug. 1996, respectively. Since 1997 he has been with the Department of Information \& Communications Engineering, Tongmyong University, Korea, where he is currently a professor. From 2000 to July 2002, he was a president of DigitalNetBank Inc. From 2005 to July 2006, he was a visiting professor in the Department of Computer and Information Engineering, Dalian Polytechnic University, China. His main research interests includes biometrics, image processing, and computer vision.

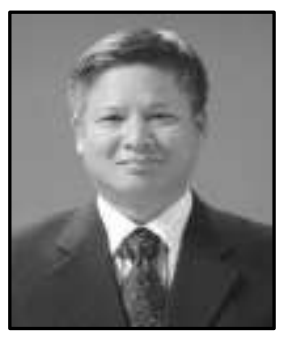

Ki-Ryong Kwon, he received the B.S., M.S., and Ph.D. degrees in electronics engineering from Kyungpook National University in 1986, 1990, and 1994 respectively. He worked at Hyundai Motor Company from 1986-1988 and at Pusan University of Foreign Language from 1996-2006. He is currently a professor in Dept. of IT Convergence \& Application Engineering at the Pukyong National University. He has researched University of Minnesota in USA on 2000-2002 with Post-Doc, and Colorado State University on 20112012 with visiting professor. He was the General President of Korea Multimedia Society from 2015-2016, also is a director of IEEE R10 Changwon section. His research interests are in the area of digital image processing, multimedia security and watermarking, bioinformatics, weather radar information processing. 
International Journal of Security and Its Applications

Vol. 11, No. 12 (2017) 